\title{
Meningioma rabdoide Grado III, en paciente pediátrico: Reporte de caso
}

Luis Orlando Rojas ${ }^{1}$, Claudia Marcela Restrepo², Andreina Martínez³ ${ }^{3}$ María Mercedes Mendoza ${ }^{4}$,

Fernando Velandia Hurtado ${ }^{5}$, Yulina Cuellar ${ }^{6}$

1 Neurocirujano, Servicio de Neurocirugía, Universidad Militar Nueva Granada, Hospital Militar Central, Bogotá, Colombia.

2 Residente de Neurocirugía, Servicio de Neurocirugía, Universidad Militar Nueva Granada, Hospital Militar Central, Bogotá, Colombia.

3 Médico y Cirujano, Universidad Militar Nueva Granada, Hospital Militar Central, Bogotá, Colombia.

$4 \quad$ Patóloga, Servicio de patología, Hospital Militar Central, Bogotá, Colombia.

5 Profesor principal de Patología y Neuropatología y miembro del Grupo de Investigación en Neurociencias (NEUROS) de la Universidad del Rosario. Bogotá, Colombia, Consultor en neuropatología de la Fundación Santafé, del Hospital Militar Central Bogotá, Colombia.

6 Residente de Patología, Servicio de Patología, Universidad Militar Nueva Granada, Hospital Militar Central, Bogotá, Colombia.

Rev. Chil. Neurocirugía 43: 141-145, 2017

\section{Resumen}

Los Meningiomas Rabdoides son una variante poco común de los meningiomas grado III según la clasificación WHO de 2016 con alto índice mitótico, invasión a tejido cerebral sano, alta tasa de recurrencia y baja expectativa de sobrevida, en esta oportunidad presentamos el caso clínico de una paciente de 23 meses de edad diagnosticada con esta patología, abarcando brevemente aspectos clínicos característicos de la patología, histopatología del diagnóstico y una revisión corta de la literatura actual sobre el tratamiento y la sobrevida de estos pacientes.

Palabras clave: Meningioma rabdoide, Tumores del sistema nervioso central, Tumor cerebral pediátrico.

\begin{abstract}
Rhabdoid meningioma are quite rare type of intracranial tumors. according to WHO classification 2016 characterized by high mitotic index, invasion to healthy brain tissue, high recurrence rate and low survival expectancy, on this occasion we present the case of a 23 months child diagnosed with this pathology, covering clinical aspects characteristic of the pathology, histopathology of the diagnosis and a short revision of the current literature on the treatment and the survival of these patients.
\end{abstract}

Keyword: Rhabdoid meningioma, intracranial tumors, pediatric brain tumors.

\section{Introducción}

Actualmente, los tumores del sistema nervioso central continúan siendo una patología poco frecuente, con incidencia ajustada por edad durante el período $2007-2011$ de 21,42 por 100.000 personas $y$, específicamente para niños y adolescentes (0 a 19 años), la incidencia es de 5,42 por cada 100.000 Según el Registro Central de Tumores Cerebrales de Estados Unidos (Central Brain Tumor Registry of the United States -CBTRUS-) uno de cada 2.000 niños es diagnosticado cada año ${ }^{1}$ sin embargo, representan el segundo tipo más común de cáncer en edad pediátrica después de los linfomas, el tumor solido más común y causan aproximadamente el $30 \%$ de las muertes oncológicas en menores de 14 años; cerca de la mitad de los tumores de sistema nervioso central en este grupo de edad son gliomas dentro de los cuales el principal es el astrocitoma pielocitico seguido de los tumores de origen embrionario ${ }^{2,15}$. Por otra parte, los me- 
ningiomas constituyen el tipo de tumor intracraneal más común en edad adulta alcanzando el $36 \%$ según CBTRUS con una incidencia aproximada de 7,61 por cada 100.000 para el 2015 y la incidencia aumenta exponencialmente con la edad, siendo dos veces más común en mujeres que en hombres para los adultos $^{3}$ aunque únicamente representan el $2 \%$ de las neoplasias primarias del SNC en la edad pediátrica.

Este subtipo tumoral se deriva de las células aracnoideas de las leptomeninges, generalmente los meningiomas son de características benignas es decir grado I según la clasificación WHO de la OMS de 2016 lo cual indica, bajo grado de compromiso a tejido cerebral sano, escasa mitosis y ausencia de características histológicas de malignidad $^{15}$, solamente entre $5-20 \%$ representan diferenciación atípica (grado II OMS) para lo cual deben cumplir al menos 3 de 5 criterios histológicos de acuerdo a la clasificación WHO 2016: necrosis, agrupamiento laminar (perdida del patrón fasciculado), nucléolos prominentes, alta celularidad, grupos de células tumorales con alto índice citoplasmático $^{15}$, y únicamente entre $1-3 \%$ son de diferenciación maligna (grado III OMS) los cuales se diagnostican histológicamente por la presencia de patrones morfológicos papilares, rabdoides y presencia de anaplasia, siendo estos últimos los que cuentan con mayor tasa de recidiva en los siguientes dos años posterior a la resección quirúrgica ${ }^{4,5}$. Lo cual representa un mal pronóstico a largo plazo para estos pacientes especialmente aquellos que son diagnosticados en los primeros años de vida.

La morfología rabdoide en los tumores hace referencia a la presencia de células con alta similitud a los rabdomioblastos (sin diferenciación clara de las células del músculo esquelético) y las características citológicas incluyen abundante citoplasma eosinófilo, núcleos eccéntricos, e inclusiones hialinas intracitoplasmaticas, clínicamente, la presencia de este patrón confiere alta tasa de malignidad al diagnóstico oncológico ${ }^{6}$.

\section{Presentación del caso}

Presentamos el caso de una paciente femenina de 23 meses de edad natural y procedente de Bogotá - Colombia sin antecedente de importancia quien ingresa por urgencias al servicio del Hospital Militar Central, con cuadro clínico de 24 horas de evolución consistente en 3 episodios eméticos en proyectil, deshidratación y deterioro progresivo del estado de conciencia, al ingreso se encuentra paciente en coma con midriasis izquierda, deshidratación grado 2 , inicialmente se interpreta como posible herniación uncal, se solicita escanografia cerebral simple (Figura $1 \mathrm{~A}$ y $B)$ donde se observa lesión ligeramente isodensa fronto parieto occipital izquierda con sangrado intralesional que ejerce importante efecto de masa desplazando la línea media $22 \mathrm{~mm}$ y pro- duce herniación del uncus. A los paraclínicos se encuentra anemia (hgb: 8,7 $\mathrm{mg} / \mathrm{dl})$, trombocitopenia $\left(123.000 \mathrm{~mm}^{3}\right.$ ) y tiempos de coagulación prolongados (tiempo de protrombina 18,2 seg, tiempo parcial de tromboplastina: 37,5 seg). Dado el estado neurológico se decide ingresar a cirugía de urgencia, realizándose drenaje del hematoma y resección tumoral hasta cuando es necesario suspender el procedimiento por inestabilidad hemodinámica. Se reseca lesión macroscópica de aspecto grisáceo, muy vascularizada, similar al tejido cerebral con sangrado fácil y muy difícil de controlar.

Durante el pos operatorio inmediato

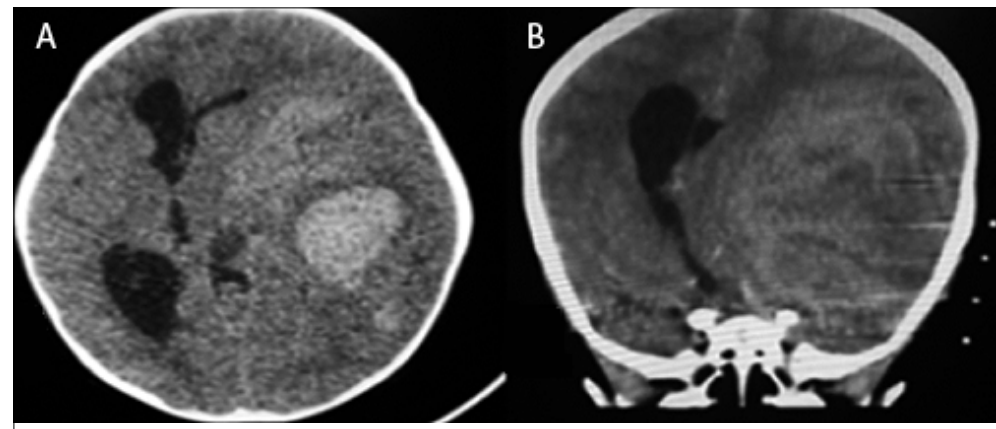

Figura 1. A) TAC, corte transversal, imagen hiperdensa en región parietoccipital izquierda; B) TAC, corte coronal, efecto de masa que desplaza la línea media.
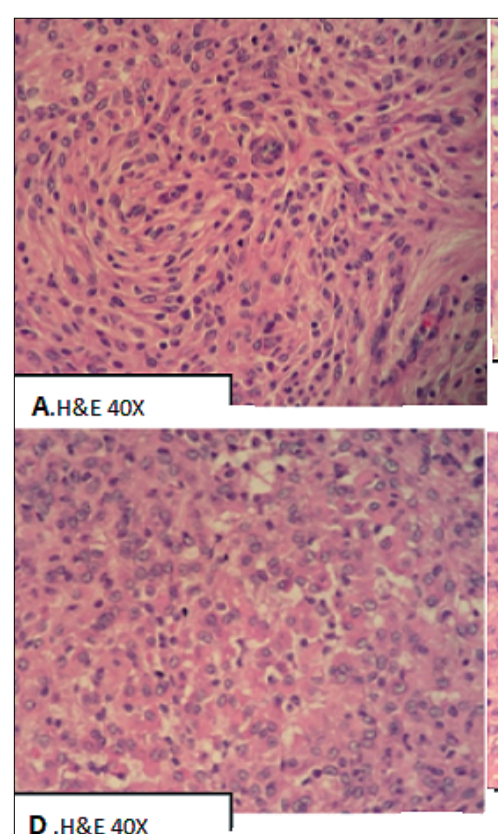

B.H\&E 40X

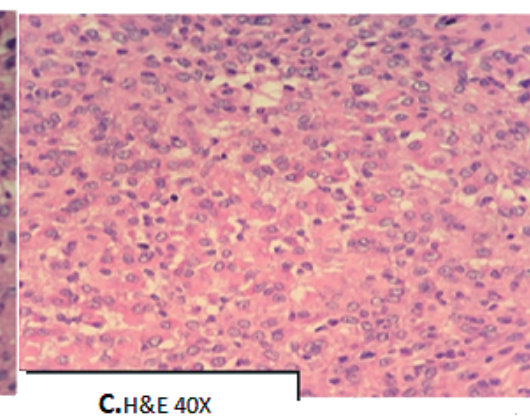

C.H\&E $40 X$

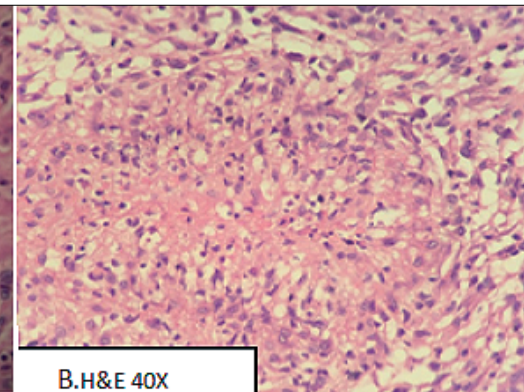

Figura 2. A,B,C,D: Neoplasia meningotelial constituida por células rabdoides, con núcleo excéntrico, con seudoinclusiones, nucléolo prominente y citoplasma eosinofilico; se aprecia aumento en el numero de mitosis, áreas de necrosis y zonas de meningioma convencional. 
se indica monitoria de presión intracraneana, una vez estabilizada hemodinamicamente se observa mejoría del estado de conciencia, paciente alerta con mejoría de anisocoria, no déficit de pares, no déficit motor ni sensitivo; sin embargo, se documentaron picos febriles, taquicardia, fluctuación en los registros de la tensión arterial y $\mathrm{PIC}$ elevadas hasta $38 \mathrm{mmHg}$, por lo cual luego de junta de unidad de cuidados intensivos pediatricos y neurocirugía y estabilizado su estado hemodinámico a las 72 horas se reinterviene. Encontrando lesión de similares características a la primera intervención quirúrgica con tejido friable, muy vascularizado y que en algunas áreas ya infiltraba el parénquima cerebral. Se hizo una resección bastante amplia, se envían muestras del tejido resecado para estudios patológicos. Nuevamente fue necesario suspender el procedimiento por solicitud de anestesiología ante la inestabilidad hemodinámica de la paciente y la severidad de la acidosis metabólica; En el pos operatorio de la segunda intervención quirúrgica la paciente evoluciona satisfactoriamente sin ningún tipo de déficit neurológico, deambulando por su habitación, no presentando nuevos episodios febriles ni de alteración en su seguimiento de signos vitales, interpreto como disfunción hipotalámica secundaria a la compresión tumoral.

El reporte final de patología sobre las muestras obtenidas indica lesión tumoral constituida por células fusiformes con nucleos ovales, algunos con aclaramiento central de la cromatina formando pseudoinclusiones dispuestas en su mayoria en un patrón arremolinado con distribución perivascular, en numerosos focos se observan células poligonales con citoplasma eosinofilo y núcleo excéntrico, se observan focos de necrosis y escasa mitosis (menos de 1 en 10 campos de alto poder) (Figura 2). La inmunohistoquímica demostró positividad para EMA, Vimentina y focalmente para Enolasa, negativa para proteína glial fibrilar S100 y Sinaptofisina, actina de músculo liso. Ki67 positivo en aproximadamente $20 \%$ de las células tumorales, progesterona positivo focal en ocasiones (Figura 3). Diagnóstico histopatológico final: Meningioma Rabdoide grado III de la OMS (2016).

Posteriormente se solicita tomografía axial computalizada de control muestra residuo tumoral y dado la patología que

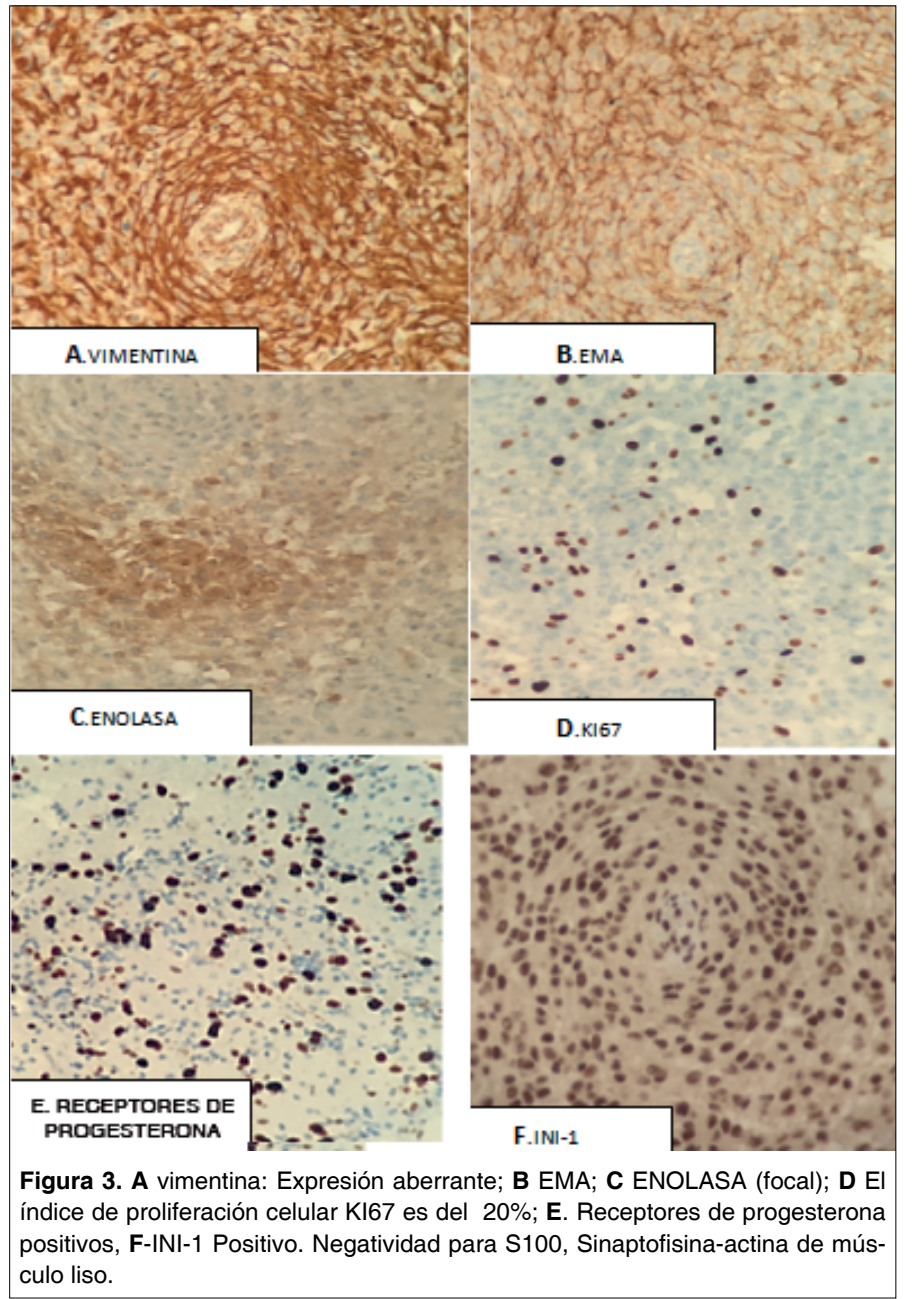

no responde al tratamiento complementario de quimio o radioterapia (no indicada en la paciente por ser menor de 3 años) se decide nueva intervención quirúrgica efectuada 45 días después y en la imagen de control pre quirúrgica se observa casi la duplicación del residuo tumoral del segundo tiempo quirúrgico (Figura 4A). En esta ocasión, se observa que la lesión infiltra ya el tejido celular, lo cual limita la resección total efectuada con neuronavegación.

En la actualidad, dos años después de la primera intervención quirúrgica, la paciente continua asistiendo a controles por el servicio de neurocirugía del Hospital Militar Central (Bogotá, Colombia) clínicamente sin evidencia de compromiso en el estado neurológico general y adecuada progresión en el neurodesarrollo, sin nuevas alteraciones del estado de conciencia y continua en manejo con quimioterapia basada en altas dosis de metotrexate por

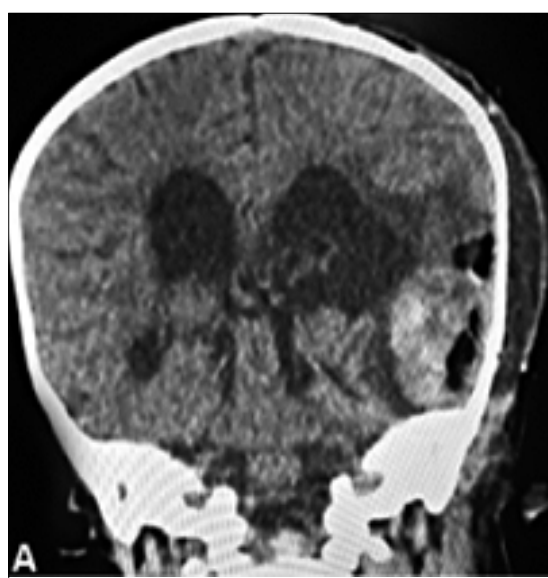

Figura 4. A) TAC, corte coronal previo a tercera intervención quirúrgica, se evidencia presencia de importante tejido tumoral residual, sin desplazamiento de línea media.

el servicio de Oncología Pediátrica de la institución. 


\section{Discusión}

Los meningiomas son neoplasias del SNC que se derivan de las células de las vellosidades aracnoideas, aunque representan un porcentaje pequeño de las neoplasias intracerebrales en edad pediátrica están asociados a mayor malignidad y tienen predilección por el sexo masculino contrario a lo que pasa en la población adulta donde es más prevalente en mujeres que en hombres ${ }^{7}$ el término "tumor rabdoide" fue introducido en 1978 por Beckwith y Palmer haciendo referencia a un subtipo tumoral renal en edad pediátrica que posteriormente se encontró en otros órganos incluyendo hígado, estómago y tiroides, entre otros, sin embargo, fue hasta 1998 cuando Kepes y luego Perry et al, describieron los hallazgos histopatológicos en una serie de pacientes con Meningiomas de comportamiento particularmente maligno, con morfología Rabdoide ${ }^{8,9}$, desde el año 2000 se añadió este subtipo a la clasificación WHO de la OMS para los tumores intracerebrales y en la más reciente actualización de 2016 se confirmó su clasificación como meningiomas grado III de acuerdo a la presencia de características histológicas de atipia más morfología rabdoide, y anaplasia ${ }^{15}$; lo cual indica un mal pronóstico para el paciente, requerimiento de manejo multidisciplinario con resección quirúrgica más terapia adyuvante y en general se habla de sobrevida estimada entre 2-3 años para pacientes adultos $^{10}$. Es importante mencionar que aunque en la mayoría de los casos se tratan de tumores de comportamiento agresivo y mal pronóstico, no todos los meningiomas rabdoides tienen el mismo grado de malignidad, de hecho para que se considere grado III la morfología rabdoide debe estar presente en más del $50 \%$ de la muestra y en aquellos en los que se evidencia únicamente la morfología rabdoide sin otros hallazgos sugestivos de malignidad se pude considerar grado II aunque este aspecto se encuentra en discusión, hasta el momento los meningiomas con escasa presencia de morfología rabdoide representan una marcada minoría ${ }^{11}$.

Actualmente, más de una década después de la primera serie de casos descrita en la literatura médica, continua siendo un subtipo tumoral poco frecuente según la revisión realizada por Abolfotoh $\mathrm{M}$ et al, en el 2012 únicamente se encontraban reportados 102 casos en la literatura, 100 de ellos intracraneales y 2 a nivel de columna ${ }^{12}$. Lo que representa un reto para todos los profesionales de la salud, desde la atención primaria del paciente que en la mayoría de los casos debuta con sintomatología neurológica de ocupación de fosa posterior que incluye cefalea intensa de larga data, vómitos en proyectil, alteración de la marcha (particularmente difícil de evaluar en pacientes pediátricos) y alteración del estado de conciencia.

Otro de los inconvenientes que se presenta con el diagnóstico de este subtipo tumoral y como es el caso que presentamos en esta oportunidad, es la dificultad anatómica que implica realizar una resección quirúrgica satisfactoria de la masa tumoral, en la mayoría de los casos los meningiomas encuentran en la región supratentorial principalmente en los senos venosos dúrales de la convexidad del cerebro, parasagital y en la región esfenoidea ${ }^{13}$ por lo tanto, son patologías que requieren craneotomías de gran envergadura y resecciones asociadas a estructuras anatómicas altamente vascularizadas y de alto impacto en la funcionalidad post operatoria del paciente, sin dejar de lado la alta posibilidad de recurrencia de células tumorales durante los primeros 12 meses lo cual indicaría una nueva intervención quirúrgica.

Por otra parte, dentro del manejo sugerido para los meningiomas grado III esta realizar resección quirúrgica seguida de qumioterapia para disminuir el riesgo de recidiva dentro de los primeros 12 meses de la resección, en los pacientes pediátricos aún no se encuentra completamente estandarizado el protocolo, principalmente asociado a los efectos adversos, hoy en día se ha demostrado que una terapia intensiva basada en alkylator más un régimen de altas dosis de metotrexate parecen tener la mayor efectividad, sin embargo, aun con el manejo adyuvante completo en la mayoría de los casos los pacientes van a requerir re-intervención quirúrgica para retirar la mayor cantidad de tejido tumoral posible ${ }^{14}$.

Consideramos que el caso que se presenta en esta ocasión es de alta relevancia médica teniendo en cuenta varios aspectos: el primero de ellos se basa en las características histopatológicas del tumor resecado, teniendo en cuenta que no se trata del más común esperado para la edad de la paciente y el escaso número de casos reportados en la literatura médica; por otro lado, la dificultad que representa el manejo neuroquirúrgico de esta patología en una paciente pediátrica, desde el método de abordaje hasta el manejo de imprevistos que se pueden presentar durante el procedimiento y, pese a los inconvenientes presentados dentro del quirófano consideramos que a largo plazo la paciente respondió de forma ampliamente satisfactoria a la resección aun cuando se trataba de un tumor hemisférico supratentorial altamente vascularizado, y sin lugar a dudas uno de los aspectos que queremos resaltar mediante la publicación de este artículo es la sobrevida que ha presentado la paciente, superando el tiempo esperado para los meningiomas grado III sin repercusiones en el neurodesarrollo para la edad durante los años siguientes, sin requerimiento de nuevas intervenciones hasta el momento y adecuado desarrollo de funciones neurológicas superiores.

\section{Conclusión}

El caso que presentamos en esta oportunidad representa una condición patología llamativa y poco usual en la población pediátrica, cursando con clínica agresiva y de rápida progresión aun en pacientes sin ningún tipo de antecedentes, que requiere manejo urgente tanto quirúrgico como médico para asegurar la sobrevida del paciente y buscando la confirmación del diagnóstico por medio de estudios patológicos, sin embargo, aun con la terapia adyuvante completa la probabilidad de requerir nuevas intervenciones quirúrgicas es muy alta lo que implica mayores riesgos para el paciente, por lo tanto, es indispensable que los profesionales de la salud estén relacionados con esta patología para que puedan sospecharla de manera oportuna y se asegure atención integral por los servicios de neurocirugía, neuropatología y oncología pediátrica en el menor tiempo posible, así como es responsabilidad de todos aquellos que tienen la oportunidad de formar parte de los servicios tratantes de pacientes con esta patología reportar estos casos buscando ampliar la literatura disponible para facilitar el estudio de temas relacionados.

Recibido: 26 de abril de 2017 Aceptado: 28 de mayo de 2017 


\section{Referencias}

1. Ostrom QT, Gittleman H, Liao P, et al. CBTRUS statistical report: primary brain and central nervous system tumors diagnosed in the United States in 2007- 2011. Neuro Oncol 2014; 16(Suppl 4): iv1-63.

2. American Cancer Society Inc., 2003. Facts and Figures. Surveillance Research, Atlanta, GA, Neurol Clin 34: (2016); 981-998.

3. Ostrom QT, Gittleman H, Liao P, et al. CBTRUS statistical report: primary brain and central nervous system tumors diagnosed in the United States in 2007- 2011. Neuro Oncol 2014; 16(Suppl 4): iv1-63.

4. Miranda Maldonado I, Larralde L, Alvarado Y, Barboza O, Ancer J. Meningioma con diferenciación rabodide. Reporte de un caso revisión de la literatura. Medicina universitaria 2010; 12 (46): 54-58.

5. Barresi V, Caffo M, Tuccari G. Classification of human meningiomas: lights, shadows, and future perspectives. J Neurosci Res. 2016 Dec; 94(12): 1604-1612.

6. Haas JE, Palmer NF, Weinberg AG, Beckwith JB. Ultrastructure of malignant rhabdoid tumor of the kidney. A distinctive renal tumor of children. Hum Pathol 1981; 12: 646-657.

7. Miranda Maldonado I, Larralde L, Alvarado Y, Barboza O, Ancer J. Meningioma con diferenciación rabodide. Reporte de un caso revisión de la literatura. Medicina universitaria 2010; 12 (46): 54-58.

8. Perry A, Scheithauer BW, Stafford SL, Abell-Aleff PC, Meyer FB. "Rhabdoid" meningioma: an aggressive variant. Am J Surg Pathol. 1998; 22(12): 1482-1490.

9. Kepes JJ, Moral LA, Wilkinson SB, Abdullah A, Llena JF. Rhabdoid transformation of tumor cells in meningiomas: A histologic indication of increased proliferative activity: Report of four cases. Am J Surg Pathol. 1998; 22: 231-238.

10. Louis DN, Ohgaki H, Wiestler OD, editors. World Health Organization Classification of Tumors of the Central Nervous System. 4th ed. Lyon:International Agency of Research on Cancer; 2007.

11. Biernat W. 2000 World Health Organization classification of tumors of the nervous system. Pol J Pathol 2000; 51: 107-114I.

12. Abolfotoh M, Tavanaiepour D, Hong C, Dunn IF, Lidov H, Al-Mefty O. Primary calcified rhabdoid meningioma of the cranio-cervical junction: A case report and review of literature. J Craniovertebr Junction Spine. 2012; 3(1): 32-37.

13. Santosh S, Bridget JJ, Lee V. Update on Meningiomas, The Oncologist 2011; 16: 1604-1613.

14. Santosh S, Bridget JJ, Lee V. Update on Meningiomas, The Oncologist 2011; 16: 1604-1613.

15. Louis DN, Perry A, Reifenberger G, von Deimling A, Figarella-Branger D, Cavenee WK, Ohgaki H, Wiestler OD, Kleihues P, Ellison DW. The 2016 World Health Organization Classification of Tumors of the Central Nervous System: a summary. Acta Neuropathol. 2016 Jun; 131(6):803-20. doi: 10.1007/s00401-016-1545-1

\section{Correspondencia a:}

Andreina Martinez Amado

Médico General y Cirujano, Hospital Militar Central, Bogotá, Colombia.

Tel: (+57) 3184299693

andreyta13ma@ hotmail.com 